\title{
Today and Future of Age-Related Macular Degeneration
}

\author{
Kang Liu and Bolin Xie \\ Department of Ophthalmology, Kunming General Hospital of PLA, Yunnan, Kunming 650038, China
}

Correspondence should be addressed to Bolin Xie, bolin_xie@tom.com

Received 27 December 2011; Accepted 29 January 2012

Academic Editors: H. Ahmadieh, B. Bui, A. Kakehashi, and W. H. Seiple

Copyright $\odot 2012$ K. Liu and B. Xie. This is an open access article distributed under the Creative Commons Attribution License, which permits unrestricted use, distribution, and reproduction in any medium, provided the original work is properly cited.

\begin{abstract}
Age-related macular degeneration (AMD) is the leading cause of blindness in people over 50 in developed countries. Understanding of the pathologic process, genetic mechanisms, and risk factors of this disease has the benefit of seeking newer and more effective treatment options. Current clinical therapy for AMD shows a dramatic change from a decade ago. Anti-VEGF drug therapy is regarded as the more effective treatment for neovascular AMD now, especially combining PDT therapy. In the future, the genetic and biochemical therapies may be the promising treatments for AMD. This paper will focus on the progress of pathology, candidate genes of AMD, risk factors, and the existing drugs or surgical therapies available, in order to present some new directions of care with the prospect of improved vision in many patients suffered from AMD.
\end{abstract}

\section{Introduction}

Age-related macular degeneration (AMD) remains one of the most severe and profound disabilities encountered in medicine, particularly due to the loss of the central vision and the high economic burden it places on patients and societies. AMD is regarded as the most primary cause of blindness, particularly irreversible blindness [1]. The world Health Organization (WHO) summarised the most recent reports which the visual impairment was caused by many common ocular diseases, it was not difficult to find that AMD is the most common cause of blindness. AMD has resulted in the physical and mental health problems of a number of the geriatric population and their families tremendously. Recent years, it was not only high morbidity of AMD in western nations but also progressive incidence in eastern Asia areas, thus, continued research will be critically needed.

There were about over one hundred years for scientists to study AMD. Hutchison and Tay published an article named "symmetrical central choroidoretinal disease occurring in senile persons" in 1875 . This may be the earliest medical literature about AMD that we can find. We reviewed the basic research towards AMD during about decade. There were three aspects should be summarized, including morphology, molecular biology, and genomics. AMD resulted from many dangerous factors, including genes and environment. With advancing age, some changes occurred in the retina, including alterations in retinal pigment epithelium (RPE) cellular size and shape, thickening of Bruch's membrane, thickening of the internal limiting membrane, and a decrease in retinal neuronal elements [2]. From a histopathologic standpoint, the initial morphological changes impacted on the photoreceptors and RPE cells, Bruch's membrane, and choriocapillaris in this multifactorial disease. The changes in the RPE and chioroid with age might provide a background for the development of AMD. An important pathological change in AMD was choroidal neovascularization (CNV). It was the defining feature of neovascular AMD, wet AMD, or exudative AMD. These new vessels growed into the subretinal space and caused classical pathological process of wet AMD [3]. Recently, the etiology of AMD kept a great many of unknown, numerous studies have suggested that genes are one of more important risk factors significantly impacted on AMD developing.

Ophthalmic genetics researches have carried out for about a decade, scientists tried to find the relationship between identification of risk factors associated with susceptibility to AMD and genes. A significant breakthrough in search of genetic contributors in AMD came in the spring of 2005. DNA sequence variant in complement factor $\mathrm{H}$ associated with AMD was found by four independent groups. They suggested that this variant, a nonsynonymous 
or disease relevant single nucleotide polymorphism (SNP), corresponds to a tyrosine to histidine polymorphism $[4,5]$. Subsequently, LRP6 and VEGF were found that they may play a role in the risk of developing AMD [6]. But identification of the underlying genes was be difficult, with both genomic screen (locational) and candidate gene (functional) approaches being used.

Currently, not only in the developed countries but also developing countries, there are about $6.5 \%$ of people over 50 years who is affected by AMD. The underlying reasons for AMD rest with race, gene, age, gender, diet, smoking, education, cardiovascular disease, and sunlight group, and so on. In order to stop the progress of this disease, molecular mechanisms underlying AMD pathogenesis should be further focused on, and the need for novel therapeutic and preventative strategies for AMD is pressing. Established therapies for neovascular AMD, like photodynamic therapy (PDT) and anti-VEGF therapies, allow stabilization or even improvement of vision. Potential future drugs are under development for advanced AMD or its prevention targeted the signal transduction cascade of different angiogenic molecules. These drugs intervene at different levels of the involved processes including the RNA production and specific protein expression as well as inflammatory, apoptotic, or metabolic processes.

In this paper, we review the histopathologic findings that define AMD, along with new molecular pathologic findings that have advanced our understanding of the molecular mech-anisms of AMD pathogenesis. Meanwhile, we aim at the current new therapies and to highlight possible future therapeutic strategies.

\section{Pathology of AMD}

The progression of AMD occurs over a few decades. Over the age of 70 , the incidence of the disease increases dramatically. AMD is a heterogeneous group of disorders and exhibites the variability of symptoms, clinical findings, and natural history observed in patients. At present, it was known that AMD affects the neural retina, photoreceptors, RPE, Bruch's membrane, and the choriocapillaris. Clinically, early AMD is characterised by large drusen, go by the name of dry AMD. With the development of this disease, RPE pigmentary changes can progress to geographic atrophy. When the CNV was found in macular region, we defined the late stage as neovascular AMD or wet AMD. Although the pathogenesis of AMD remains greatly unclear, a complex interaction of genetic and environmental factors is thought to exist.

2.1. Early Stage AMD. Pathological changes of early AMD include the Bruch's membrane thickening and abnormal architecture, lipofuscin accumulation in the RPE, and drusen formation beneath the RPE in Bruch's membrane.

The earliest pathological changes are characterized by basal laminar deposits (BlamD) and basal linear deposits (BlinL). BlamD of AMD was located between the basement membrane of the RPE and its plasma membrane consists of basement membrane proteins and long-spacing collagen [79]. Subsequent reports confirmed that BlamD was the most prevalent histopathologic finding in early AMD eyes used as a marker for AMD grading [10], its thickness correlating well with the degree of RPE degeneration, photoreceptor fallout, and vision loss $[11,12]$. The early BLamD clinically displays a normal fundus, and the late BLamD correlates most closely with clinical pigment abnormalities. It is the quantity and sites of membranous debris accumulation that appear to determine whether the disease develops pigment changes only or follows the alternative pathway of soft drusen formation with its attendant greater risk of CNV [13].

BlinL is made of vesicular material. It located in the inner collagenous region of Bruch's membrane. BlinL and BlamD are important positive associations with $\mathrm{CNV}$, disciform scarring, and visual loss [11].

In early AMD, drusen are most frequently found as clusters within the macular region. If drusen diameter exceeds $25 \mathrm{~mm}$, it may be visible ophthalmoscopically as yellowwhite deposits [5]. Many laboratories studying these dumbbell shaped deposits have shown that drusen was composed of glycoproteins and lipids and accumulates in extracellular subretina often be located between the RPE basal lamina and Bruch's membrane. Clinically, drusen is classified morphologically either as hard or soft. Hard drusen exhibits some small, yellowish, and punctuated deposits. But it is not considered a particularly important risk factor for the development of AMD, especially drusen that is few in number and hard in quality. Hard drusen exists in common elderly patients without AMD also. In contrast, soft drusen presents a more diffuse, paler, and larger appearance, perhaps with "fuzzy" or blurry edges. The large, bilateral, and/or numerous soft drusen is significant risk factors for development of late stage AMD. Dynamic remodelling processes of drusen resorption and new drusen formation are maybe a useful marker of disease activity [14].

Although AMD is not a classic inflammatory disease, inflammation has been found to have an important role in disease pathogenesis and progression. Hageman et al. suggest that immune system may be involved in AMD pathogenesis through studies about inflammation-related molecules in drusen [15-17]. A recent research pointed out that recruitment of leukocytes and activation of the complement cascade occurred in mouse RPE and choroid during the process of age-related changes [18]. Hence, some researches targeted some specific inflammatory molecules, complement components, lipids, lipoproteins B and E, and glycoproteins, which are common constituents of drusen, in an attempt to better understand and treat AMD. Therefore, some reports deemed that anti-inflammatory agents such as corticosteroids, nonsteroidal anti-inflammatory drugs (NSAIDs), and immunosuppressive agents mighty provide an adjunct or alternative mechanism to suppress the inflammatory processes driving AMD progression [19].

2.2. Late Stage AMD. Late stage dry AMD is clinically characterized by geographic atrophy, which shows roughly oval areas of hypopigmentation and is often the consequence of RPE cell loss. Death of RPE cells led to the gradual degeneration of nearby photoreceptors. Subsequently, the retina became thin, going with progressive vision impairment. At 
the periphery of these hypopigmented areas, hyperpigmentary changes associated with compensatory RPE cell proliferation [20].

Late stage wet AMD attributes to choroidal neovascularization. Thickening of RPE basal lamina, decreasing of number of RPE cells and fundus structure impairment indicate the growth of choroidal new blood vessels [9-11]. Subretinal neovascular fibrous tissue can be found at macular area. The neovascularization has two different patterns: firstly new capillary vessels sprout from the choroidal vessels and penetrate through barrier formed by Bruch's membrane. Secondly, new vessels derive mainly from the retinal circulation, and they extend outward into the subretinal space. These vessels develop sometimes anastomose with the choroid-derived vessels [21-23]. It is called retinal angiomatous proliferation (RAP). However, RAP is much less frequently than CNV.

\section{Candidate Gene of AMD}

The AMD is greatly influenced by genetic factors in the pathogenesis, and genetic mechanisms underlying this disease are complex. Studies with environmental, genetic, and systemic health differences in development of AMD would provide a new understanding of the dynamic interplay between genes and environment. Researches have indicated a numer of AMD-associated genes in mechanistic pathways related to DNA repair, complement system activation, microglial recruitment, inflammation, neovascularization, and extracellular matrix function [24].

In 2005, independent research groups across the United States published a breakthrough identifying a DNA sequence variant in complement factor $\mathrm{H}$ that is associated with AMD. They found that single nucleotide polymorphism corresponding to a tyrosine to histidine polymorphism at position 402 which profoundly raise the odds for developing AMD $[25,26]$. great many previous studies about genes have focused on SNP. Apparently, SNP and AMD have highly significant statistical associations, but they cannot account for the entire genetic component of the disease. Besides, there are some questions which whether the identified SNP is causally related to deviant gene function or whether it is plainly genetic markers. Conrad et al. reported another type of genetic structural variation called copy number variation, which has been known as an authentic source of phenotypic variation in human populations and be suitable for quantitative characterization [27]. But these methods did not find strong evidence related with AMD-associated genes.

So far, we found the main candidate genes related with AMD as follows: fibulin 5, CST3, CX3CR, TLR4, VEGF, LRP6, MMPs, HLA family of genes, and CFH.

Fibulin 5 is a $52-\mathrm{kDa}$ calcium-binding epidermal growth factor (cbEGF-) rich extracellular matrix protein that is essential for the formation of elastic tissues. Ten fibulin 5 sequence variants have been associated with AMD and two other fibulin 5 mutations caused autosomal-recessive cutis laxa. Fibulin-5 was localized to BlamD beneath the RPE as well as some small drusen [28-30].
Cystatin $\mathrm{C}$ is a cysteine protease inhibitor that regulates the activity of cathepsin $S$, a protease with central regulatory functions in retinal pigment epithelial cells. Genotyping data suggest an increased susceptibility for AMD in CST3 B/B homozygotes. CST3 B may be a recessive risk allele [31].

CX3CR1 is expressed on retinal microglia cells and plays an important role in the activation and migration of the microglia [32]. Some reports deem that CX3CR1 is associated with wet AMD in Han Chinese and Caucasian population [33].

TLR4 (toll-like receptor 4) genetic variant alters the extracellular domain of the receptor, which interrupts the signaling transduction cascade and interferes with the expression of genes such as TNF-a, IL-1, IL-6, monocyte chemoattractant protein-1 (MCP-1 or CCL2), and its cognate receptor C-C chemokine receptor-2 (CCR2) [34]. Some researches suggest TLR4 as a candidate gene for AMD [35].

VEGF (vascular endothelial growth factor) is a major player in the control of angiogenesis and has a strong role to play in neovascularization. Increased expression of VEGF gene in the RPE and in the outer nuclear layer is associated with wet AMD [36, 37].

LRP6 (low-density lipoprotein receptor-related protein 6) is involved in vasculature remodeling pathways. Some data show LRP6 may play a role in the risk of developing AMD [6].

MMP (matrix metalloproteinase) is zinc-dependent endopeptidases; other family members are adamalysins, serralysins, and astacins. The MMPs belong to a larger family of proteases known as the metzincin superfamily. MMPs are also thought to play a major role on cell behaviors such as cell proliferation, migration (adhesion/dispersion), differentiation, angiogenesis, apoptosis, and host defense. Reduction in the levels of activated MMPs -2 and -9 may be responsible for impaired matrix degradation of Bruch's membrane leading to the pathology associated with AMD [38].

HLA (human leukocyte antigen) complex helps the immune system distinguish the body's own proteins from proteins made by foreign invaders. The HLA-Cw0701 allele, KIR haplotype AA, and HLA-B27 are associated with AMD [28, $29,39]$.

$\mathrm{CFH}$ (Complement Factor H) mediates a number of essential biological functions that participate in host defense against infection, initiation of the inflammatory reaction, processing, and clearance of immune complexes, and regulation of the immune response. CFH rs3753394, rs800292, rs1410996, rs800292, rs1061170, and rs1329428 that are the AMD-associated single-nucleotide polymorphisms showed a significant association with wet AMD [33, 40-42].

\section{Current AMD Clinical Researches, Therapies, and Prevention}

4.1. Epidemiology. Many population-based epidemiologic studies examined age-related macular degeneration (Table 1) [43-49]. Recently, a USA nationally representative, population-based, and cross-sectional study involving a total of 5553 persons reported that the estimated prevalence of 
TABle 1: Epidemiological Studies of Age-related Macular Degeration (early AMD and exudative AMD): distribution by age groups (\%), (percentages for exudative AMD are given in parentheses).

\begin{tabular}{|c|c|c|c|c|}
\hline Study/age & $50-59$ & $60-69$ & $70-79$ & Over 80 \\
\hline Beaver Dam (USA) 4756 patients & $16.7(0.5)$ & $25.3(1.4)$ & $41.7(6.9)$ & $48.7(13.5)$ \\
\hline $\begin{array}{l}\text { Proyecto VER (Hispanic people in Arizona) } \\
2780 \text { patients }\end{array}$ & $19.8(0.1)$ & $27.7(0.5)$ & $41.3(0.8)$ & $54.1(4.3)$ \\
\hline Rotterdam (Netherlands) 6411 patients & $2.5(0.1)$ & $9.9(0.7)$ & $16.7(3.2)$ & $29.8(11.6)$ \\
\hline Blue Mountains (Australia) 3585 patients & $2.8(0.2)$ & $9.2(0.7)$ & $20.9(5.4)$ & $46.6(18.5)$ \\
\hline Reykjavik eye study (Iceland) 1021 patients & $0.3(0.0)$ & $1.2(0.0)$ & $5.3(0.5)$ & $25(9.8)$ \\
\hline Beijing eye study (China) 4376 patients & \multicolumn{4}{|c|}{$1.4(0.1)^{*}$} \\
\hline
\end{tabular}

${ }^{*}$ The Beijing Eye Study are not classified by age.

any $\mathrm{AMD}$ and late $\mathrm{AMD}$, was respectively, 6.5\%, $0.8 \%$ in USA population aged 40 years and older. But there is a significantly lower prevalence of any AMD in non-Hispanic black persons aged 60 years and older [50]. Another population-based cross-sectional study among elderly residents in Northeast Italy, 1162 randomly selected subjects aged 61 years or more were estimated to affect $62.7 \%$ of the whole population. It is not difficult to demonstrate that early AMD often be found over 50 ages. With advancing age, the prevalence of late AMD gradually increases. There are different prevalences in different area of the world.

In our views, more researches should necessary understand the underlying reasons for the different patterns of presentation of early and late AMD among race, genes, age, gender, diet, smoking, education, cardiovascular disease, and sunlight groups in order to explore the predictive value of early AMD to stop the progress of this disease in different populations.

4.2. Risk Factors. Great many risk factors have been reported to associate with AMD, such as age, iris color, cataract/cataract surgery, cardiovascular factor, hypertension, diabetes, arthritis, diet, fat, drug, gene, smoking, and family history of AMD. But the evidence and strength of association are variable. Recently, a systematic review, prospective and cross-sectional studies, involving 113,780 persons with 17,236 cases of late AMD, estimated the association between late AMD and risk factors [51]. These results of meta-analyses show that risk factors of late AMD with strong and consistent associations were increasing age, current cigarette smoking, previous cataract surgery, and a family history of AMD; moderate associations were higher body mass index, history of cardiovascular disease, hypertension, and higher plasma fibrinogen; weaker associations were gender, ethnicity, diabetes, iris colour, history of cerebrovascular disease, serum total and HDL cholesterol, and triglyceride levels.

4.3. Therapies. We review current therapeutic methods and discuss possible future strategies about AMD in this part. Dry AMD possibly translates into wet AMD at every phase in developing AMD. Especially, the neovascular exsudative form of AMD may result in severe sight loss. Although therapies for AMD were limited to some extent, new therapies have emerged for both the nonexudative and exudative
AMD, which have improved prognostic outcomes. These treatments include nutritional supplementation, antioxidant prophylaxis, intravitreal injection of medications that inhibit aberrant vascular proliferation, PDT, and transpupillary thermotherapy (TTT). However, monotherapy for AMD, especially wet AMD, produces very little effect currently. Thus, choosing of the optimal therapeutic schedule is a burning question. Presently, combination of different therapies is the best therapeutic plan for doctor to select, but method of combined treatment and consolidation treatment of followup remains a problem to be solved for wet AMD.

4.3.1. Noninvasive Therapies. Antioxidant therapy, particularly in nonexudative age-related macular degeneration, may plays a role in minimizing disease progression. The Age-Related Eye Disease Study (AREDS), a multicenter, doublemasked, randomized, controlled clinical trial revealed that oral supplementation of a combination of vitamin C, vitamin $\mathrm{E}$, beta-carotene, zinc oxide, and cupric oxide in patients with advanced AMD in one eye had a $25 \%$ relative risk reduction of developing advanced AMD in the other eye. The relative risk of vision loss of three or more lines was reduced by $19 \%$ [34]. However, there was no evidence to support that vitamin supplementation is good for patients with early nonexudative AMD or active exudative AMD in both eyes. Surprisedly, the research of the Eye Disease Case-Control Study Group demonstrated that increased intake of vegetable fat, mono- and polyunsaturated fatty acids, and linoleic acid has also been associated with an increased risk for AMD [52]. Conversely, higher intaking of fish, nuts, and dark green leafy vegetables has been associated with a lower risk for AMD [35].

TTT is a brachytherapy which uses of a long-pulse, $810 \mathrm{~nm}$ near-infrared laser irradiation. TTT can produce approximately $10^{\circ} \mathrm{C}$ for typical clinical parameters used in $\mathrm{CNV}$ treatment. But, the treatment effectiveness of TTT is uncertain, and it has complications, with posttreatment haemorrhage and macular infarction having been reported [28, 39]. It is not generally used worldwide now.

4.3.2. Invasive Therapies. PDT is relatively effective invasive therapy for exudative AMD. The evolution of subfoveal choroidal neovascularization was more than that of juxtafoveal choroidal neovascularization after PDT treatment. 
Owing to the former, it grew quickly toward the fovea and visual acuity loss was greater. PDT is a safe, long-term treatment for exudative age-related macular degeneration [53], but it is not definitive because this treatment cannot stop the initial growth of the choroidal neovascularization lesion [54].

Intravitreal ranibizumab and bevacizumab monotherapy were regarded as the more effective treatment for neovascular AMD now, they go by the name of antivascular endothelial growth factor (anti-VEGF) therapy.

Bevacizumab is a full-length monoclonal antibody that binds to and blocks the action of all VEGF isoforms. Numerous studies towards intravitreal bevacizumab have reported its efficacy for neovascular AMD and low rates of treatment related complications [55-57].

Ranibizumab is a humanized antigen-binding fragment targeted all isoforms of VEGF-A. At present, clinically, it had been approved for the treatment of all subfoveal CNV subtypes secondary to AMD $[58,59]$. Researches pointed out that patients who initially received three injections of $0.5 \mathrm{mg}$ ranibizumab intravitreously approximately 4-6 weeks apart may improve visual acuity [59].

One study towards intravitreal bevacizumab (Avastin) versus ranibizumab (Lucentis) demonstrated that the bevacizumab had too many methodological limitations to rule out any major safety concerns, and higher evidences from ranibizumab trials hinted signals for an increased ocular and systemic vascular and haemorrhagic risk which warrants further investigation [60].

The advent of anti-VEGF therapy was revolutionary in the treatment of wet AMD. But the pathophysiology of CNV involved both angiogenesis and vasculogenesis $[29,30]$. However, the effect of anti-VEGF therapy was limited to the angiogenic component of CNV. Evidence suggested that the treatment response may diminish as maturation of the $\mathrm{CNV}$ occurs $[31,32]$. In contrast, PDT therapy was possessed of the different mechanism to treatment CNV compared with anti-VEGF therapy, and it induced the occlusion of microvasculature within a $\mathrm{CNV}$ lesion. Therefore, in terms of prevalent views, main combining therapy incorporating PDT therapy and anti-VEGF therapy may be beneficial to yielding longer treatment-free intervals and requiring fewer intravitreal injections [53]. And the combination therapy may represent an alternative treatment approach and randomized multicenter clinical trials are ongoing [61].

4.3.3. Surgical Intervene. Patients with wet AMD often suffer from large submacular hemorrhage, a major source of severe vision loss. With thickness of submacular hemorrhages, subretinal fibrosis, and widespread atrophy will occur in the retina. There are two options for the displacement of submacular hemorrhage. One is injection of an expansile gas in the vitreous cavity with or without simultaneous intravitreal injecting of tissue plasminogen activator (tPA), another is removing the vitreous gel and replacing it with expansile gas with or without intravitreal or subretinal injection of tPA. But these techniques applied to small patients $[62,63]$. And the average final visual acuity of those patients in studies was poor, although the visual acuity improved transitorily after operation. Retrospective meta-analysis of removal of subretinal hemorrhage showed only modest improvement in visual acuity with a $25 \%$ recurrence rate [64]. The failure of surgical intervention attributed to largely macular atrophy after excision of CNV or evacuation of subretinal hemorrhage, which was unavoidable excise the RPE.

4.3.4. Possible New Treatments. At present, although current therapies for CNV may in some cases stop disease progression, but by and lage they do not successfully reverse visual loss in patients with AMD [65]. Significant progress has been made in the field of gene transfer to the eye, which now allows researchers to stably express nucleic acids in the different cell types that make up the eye. Such advances, which have been made primarily in the retinal degeneration field, may now be applied to provide a solution for the multitude of patients losing vision to ocular neovascularization. The mechanism of CNV is distinct and is not completely understood, they both involve an imbalance between pro- and antiangiogenic factors that leads to pathologic neovascularization [66]. The future of treating AMD appears promising particularly as we gain further insights into the genetic and biochemical pathways of the disease [66].

The development of a gene therapy regimen would offer not only the prospect of local delivery, but also local production of the antiangiogenic protein for a prolonged period of time. The strategy for treating any disease due to loss of function of a single gene is straightforward once the gene and the appropriate cell targets have been identified. There are now several different vectors available which have characteristics suitable for delivering large or small genes in a stable fashion to a variety of ocular cell types [67]. There are considerably more challenges in developing a treatment for diseases for which particular target stages/genes are ill defined. Thus, the primary challenge in developing gene therapy for $\mathrm{CNV}$ is not how to deliver the therapeutic gene but, rather, to identify the optimal molecule to deliver.

Pigment epithelium-derived factor (PEDF) is a potent inhibitor of neovascularization [68]. Data have led researchers in the field to pursue the rescue of animal models of CNV with PEDF gene transfers to establish a therapeutic proof in principle $[69,70]$.

Encouraging results have been reported with the use of the adeno-associated virus (AAV) 2-sFLT01, a vector containing the vascular endothelial growth factor receptor, angiostatin, and most recently, pigment epithelium-derived factor (PEDF). AAV that expresses a modified soluble Flt1 receptor designed to neutralize the proangiogenic activities of VEGF is likely to treatment of AMD via an intravitreal injection, owing to well-toleration, localization, and capability of long-term expression of AAV2-sFLT01 [71]. VEGF soluble receptor gene transfection can inhibit subretinal neovascularization (SRN). This method will contribute to future gene therapy for AMD [72].

A soluble form of the VEGF receptor Fms-like tyrosine kinase (sFlt-1) has been shown to bind VEGF and blocks its signaling pathway [73]. 
Moreover, AMD is a highly multifactorial disease likely caused by individual-specific combinations of etiological and risk factors that affect not only the disease itself but also its response to various treatment modalities. Thus, future studies on the genetic and pathology of AMD might help doctors develop effective therapies and ultimately improve quality of life for AMD patient.

\section{Summary}

AMD exhibited clinically loss of central vision and pathologically the accumulation of drusen, and RPE degeneration, photoreceptor atrophy and in some cases with CNV. Metaanalyses demonstrated that the current model of pathogenesis likely involves age, iris color, cataract/cataract surgery, cardiovascular factors, hypertension, diabetes, arthritis, diet, fat, drug, gene, smoking, and family history of AMD. The retina and RPE with progressive oxidative damage bring structural and biochemical changes that promote inflammation and angiogenesis. Although researchers know about some finite pathogenesis of AMD, the etiology and pathogenesis of the disease remain mainly unclear. Treatment options for different stage of AMD are similarly limited. So concomitant novel preventative and therapeutic strategies are necessary.

Oxidative stress, inflammation, lipid metabolism, and angiogenesis play significant roles on the molecular pathology of AMD in recent researches. The retina and macula in particular are highly impacted on by the raised oxygen tension, high metabolic activity, optical radiation, and oxidizing processes of photoreceptor phagocytosis. Meta-analyses demonstrated that increased risks consistently associated with cigarette smoking, genetics, and a potent oxidizing stress and decreased risk associated with higher intake of fish, nuts, and dark green leafy vegetables.

Although AMD is not a classic inflammatory disease, pathology of AMD shows signs of chronic inflammatory damage, including modest infiltration of some inflammatory cell and existence of inflammatory factors. The newest research findings prove a genetic link of the complement system. Compared to the scenario with complement, the definite role of some inflammatory cell, such as macrophages and microglia in AMD pathogenesis, is protective and harmful, it lacks of clear.

Recent experiment of animal model and clinic of AMD towards VEGF suggested that a potential role of VEGF was a key angiogenic factor in the development of $\mathrm{CNV}$ in late stage AMD, and more researchers suggested that polymorphisms in the VEGF gene might bring about risk of AMD and effect on anti-VEGF therapy. Further investigations of the gene profiles of AMD patients may play important role to anticipate different therapeutic strategies. Potentially, genomics will guide new therapy regimes and fundamentally change the therapy and prognosis of AMD. From our view, along with our understanding accumulated from the field of molecular epidemiology, genomics, and pathology of AMD, future studies on the molecular pathology of AMD will help us gain further insights into the specific molecular signatures of AMD and ultimately find out an optimal way to cure patients of AMD.

In our opinion, the future genetic tests should explain the correlation between the individual genetic risk and suffering from late AMD, in order to interfere in the progress of AMD early. Accordingly, more intensive/combination stratifies could be used with patients according to their special genotype. The gene therapy should be promising to treat all AMD.

\section{Abbreviations}

AMD: Age-related macular degeneration;

AAV: Adeno-associated virus;

BlamD: Basal laminar deposits;

BlinL: Basal linear deposits;

CFH: Complement factor $\mathrm{H}$;

CNV: Choroidal neovascularization;

HLA: Human leukocyte antigen;

LRP6: Low-density lipoprotein receptor-related protein 6;

MMP: Matrix metalloproteinase;

PDT: Photodynamic therapy;

RAP: Retinal angiomatous proliferation;

RPE: Retinal pigment epithelium;

SNP: Single nucleotide polymorphism;

TLR4: Toll-like receptor 4;

tPA: Tissue plasminogen activator;

TTT: Transpupillary thermotherapy;

VEGF: Vascular endothelial growth factor.

\section{Acknowledgment}

The authors thank Yu Geng for suggestions and discussions on the paper.

\section{References}

[1] K. M. Gehrs, D. H. Anderson, L. V. Johnson, and G. S. Hageman, "Age-related macular degeneration-emerging pathogenetic and therapeutic concepts," Annals of Medicine, vol. 38, no. 7, pp. 450-471, 2006.

[2] R. H. Rosa, B. M. Glaser, Z. De la Cruz, and W. R. Green, "Clinicopathologic correlation of an untreated macular hole and a macular hole treated by vitrectomy, transforming growth factor- $\beta 2$, and gas tamponade," American Journal of Ophthalmology, vol. 122, no. 6, pp. 853-863, 1996.

[3] W. R. Green, "Histopathology of age-related macular degeneration," Molecular Vision, vol. 5, p. 27, 1999.

[4] P. E. Rakoczy, S. H. Sarks, N. Daw, and I. J. Constable, "Distribution of cathepsin D in human eyes with or without agerelated maculopathy," Experimental Eye Research, vol. 69, no. 4, pp. 367-374, 1999.

[5] S. H. Sarks, J. J. Arnold, M. C. Killingsworth, and J. P. Sarks, "Early drusen formation in the normal and aging eye and their relation to age related maculopathy: a clinicopathological study," British Journal of Ophthalmology, vol. 83, no. 3, pp. 358-368, 1999.

[6] J. L. Haines, N. Schnetz-Boutaud, S. Schmidt et al., "Functional candidate genes in age-related macular degeneration: significant association with VEGF, VLDLR, and LRP6," Investigative 
Ophthalmology and Visual Science, vol. 47, no. 1, pp. 329-335, 2006.

[7] K. U. Loffler and W. R. Lee, "Basal linear deposit in the human macula," Graefe's Archive for Clinical and Experimental Ophthalmology, vol. 224, no. 6, pp. 493-501, 1986.

[8] G. E. Marshall, A. G. P. Konstas, G. G. Reid, J. G. Edwards, and W. R. Lee, "Type IV collagen and laminin in Bruch's membrane and basal linear deposit in the human macula," British Journal of Ophthalmology, vol. 76, no. 10, pp. 607-614, 1992.

[9] T. L. Van der Schaft, C. M. Mooy, W. C. De Bruijn, and P. T. V. M. de Jong, "Early stages of age-related macular degeneration: an immunofluorescence and electron microscopy study," British Journal of Ophthalmology, vol. 77, no. 10, pp. 657-661, 1993.

[10] S. H. Sarks, "Ageing and degeneration in the macular region: a clinico pathological study," British Journal of Ophthalmology, vol. 60, no. 5, pp. 324-341, 1976.

[11] W. R. Green and C. Enger, "Age-related macular degeneration histopathologic studies: the 1992 Lorenz E. Zimmerman lecture," Ophthalmology, vol. 100, no. 10, pp. 1519-1535, 1993.

[12] C. W. Spraul, G. E. Lang, H. E. Grossniklaus, and G. K. Lang, "Histologic and morphometric analysis of the choroid, Bruch's membrane, and retinal pigment epithelium in postmortem eyes with age-related macular degeneration and histologic examination of surgically excised choroidal neovascular membranes," Survey of Ophthalmology, vol. 44, no. 2, pp. S10-S32, 1999.

[13] S. Sarks, S. Cherepanoff, M. Killingsworth, and J. Sarks, "Relationship of basal laminar deposit and membranous debris to the clinical presentation of early age-related macular degeneration," Investigative Ophthalmology and Visual Science, vol. 48, no. 3, pp. 968-977, 2007.

[14] R. T. Smith, M. A. Sohrab, N. Pumariega et al., "Dynamic soft drusen remodelling in age-related macular degeneration," British Journal of Ophthalmology, vol. 94, no. 12, pp. 1618$1623,2010$.

[15] G. S. Hageman, P. J. Luthert, N. H. Victor Chong, L. V. Johnson, D. H. Anderson, and R. F. Mullins, "An integrated hypothesis that considers drusen as biomarkers of immunemediated processes at the RPE-Bruch's membrane interface in aging and age-related macular degeneration," Progress in Retinal and Eye Research, vol. 20, no. 6, pp. 705-732, 2001.

[16] J. W. Crabb, M. Miyagi, X. Gu et al., "Drusen proteome analysis: an approach to the etiology of age-related macular degeneration," Proceedings of the National Academy of Sciences of the United States of America, vol. 99, no. 23, pp. 14682-14687, 2002.

[17] R. F. Mullins, S. R. Russell, D. H. Anderson, and G. S. Hageman, "Drusen associated with aging and age-related macular degeneration contain proteins common to extracellular deposits associated with atherosclerosis, elastosis, amyloidosis, and dense deposit disease," FASEB Journal, vol. 14, no. 7, pp. 835-846, 2000.

[18] H. Chen, B. Liu, T. J. Lukas, and A. H. Neufeld, "The aged retinal pigment epithelium/choroid: a potential substratum for the pathogenesis of age-related macular degeneration," PLoS ONE, vol. 3, no. 6, Article ID e2339, 2008.

[19] Y. Wang, V. M. Wang, and C. C. Chan, "The role of anti-inflammatory agents in age-related macular degeneration (amd) treatment," Eye, vol. 25, no. 2, pp. 127-139, 2011.

[20] P. T.V.M. De Jong, "Age-related macular degeneration," New England Journal of Medicine, vol. 355, no. 14, pp. 1474-1485, 2006.
[21] R. Brancato, U. Introini, L. Pierro et al., "Optical coherence tomography (OCT) in retinal angiomatous proliferation (RAP)," European Journal of Ophthalmology, vol. 12, no. 6, pp. 467-472, 2002.

[22] M. C. Donati, G. Carifi, G. Virgili, and U. Menchini, "Retinal angiomatous proliferation: association with clinical and angiographic features," Ophthalmologica, vol. 220, no. 1, pp. 31-36, 2005.

[23] M. A. Hunter, M. T. Dunbar, and P. J. Rosenfeld, "Retinal angiomatous proliferation: clinical characteristics and treatment options," Optometry, vol. 75, no. 9, pp. 577-588, 2004.

[24] X. Ding, M. Patel, and C. C. Chan, "Molecular pathology of age-related macular degeneration," Progress in Retinal and Eye Research, vol. 28, no. 1, pp. 1-18, 2009.

[25] S. Zareparsi, K. E. H. Branham, M. Li et al., "Strong association of the $\mathrm{Y} 402 \mathrm{H}$ variant in complement factor $\mathrm{H}$ at $1 \mathrm{q} 32$ with susceptibility to age-related macular degeneration," American Journal of Human Genetics, vol. 77, no. 1, pp. 149-153, 2005.

[26] Y. P. Conley, A. Thalamuthu, J. Jakobsdottir et al., "Candidate gene analysis suggests a role for fatty acid biosynthesis and regulation of the complement system in the etiology of agerelated maculopathy," Human Molecular Genetics, vol. 14, no. 14, pp. 1991-2002, 2005.

[27] D. F. Conrad, D. Pinto, R. Redon et al., "Origins and functional impact of copy number variation in the human genome," Nature, vol. 464, no. 7289, pp. 704-712, 2010.

[28] R. M. Ahuja, J. D. Benner, J. C. Schwartz, J. W. Butler, and S. M. Steidl, "Efficacy of transpupillary thermotherapy (TTT) in the treatment of occult subfoveal choroidal neovascularization in age-related macular degeneration," Seminars in Ophthalmology, vol. 16, no. 2, pp. 81-85, 2001.

[29] M. A. Zarbin, "Current concepts in the pathogenesis of agerelated macular degeneration," Archives of Ophthalmology, vol. 122, no. 4, pp. 598-614, 2004.

[30] D. Kent and C. Sheridan, "Choroidal neovascularization: a wound healing perspective," Molecular Vision, vol. 9, pp. 747755, 2003.

[31] J. Bradley, M. Ju, and G. S. Robinson, "Combination therapy for the treatment of ocular neovascularization," Angiogenesis, vol. 10, no. 2, pp. 141-148, 2007.

[32] G. Bergers, S. Song, N. Meyer-Morse, E. Bergsland, and D. Hanahan, "Benefits of targeting both pericytes and endothelial cells in the tumor vasculature with kinase inhibitors," Journal of Clinical Investigation, vol. 111, no. 9, pp. 1287-1295, 2003.

[33] X. Yang, J. Hu, J. Zhang, and H. Guan, "Polymorphisms in CFH, HTRA1 and CX3CR1 confer risk to exudative agerelated macular degeneration in Han Chinese," British Journal of Ophthalmology, vol. 94, no. 9, pp. 1211-1214, 2010.

[34] A. Kassoff, J. Kassoff, J. Buehler et al., "A randomized, placebocontrolled, clinical trial of high-dose supplementation with vitamins $\mathrm{C}$ and $\mathrm{E}$, beta carotene, and zinc for age-related macular degeneration and vision loss: AREDS report no. 8," Archives of Ophthalmology, vol. 119, no. 10, pp. 1417-1436, 2001.

[35] J. M. Seddon, J. Cote, and B. Rosner, "Progression of agerelated macular degeneration: association with dietary fat, transunsaturated fat, nuts, and fish intake," Archives of Ophthalmology, vol. 121, no. 12, pp. 1728-1737, 2003.

[36] M. Ip, A. Kroll, and E. Reichel, "Transpupillary thermotherapy," Seminars in Ophthalmology, vol. 14, no. 1, pp. 11-18, 1999. 
[37] E. Reichel, A. M. Berrocal, M. Ip et al., "Transpupillary thermotherapy of occult subfoveal choroidal neovascularization in patients with age-related macular degeneration," Ophthalmology, vol. 106, no. 10, pp. 1908-1914, 1999.

[38] A. A. Hussain, Y. Lee, J. J. Zhang, and J. Marshall, "Disturbed matrix metalloproteinase activity of Bruch's membrane in agerelated macular degeneration," Investigative Ophthalmology \& Visual Science, vol. 52, no. 7, pp. 4459-4466, 2011.

[39] R. S. B. Newsom, J. C. McAlister, M. Saeed, and J. D. A. McHugh, "Transpupillary thermotherapy (TTT) for the treatment of choroidal neovascularisation," British Journal of Ophthalmology, vol. 85, no. 2, pp. 173-178, 2001.

[40] Y. Yu, T. R. Bhangale, J. Fagerness et al., "Common variants near FRK/COL10A1 and VEGFA are associated with advanced age-related macular degeneration," Human Molecular Genetics, vol. 20, no. 18, pp. 3699-3709, 2011.

[41] X. Liu, P. Zhao, S. Tang et al., "Association study of complement factor $\mathrm{H}, \mathrm{C} 2, \mathrm{CFB}$, and $\mathrm{C} 3$ and age-related macular degeneration in a Han Chinese population," Retina, vol. 30, no. 8, pp. 1177-1184, 2010.

[42] L. Dong, Y. Qu, H. Jiang et al., "Correlation of complement factor $\mathrm{H}$ gene polymorphisms with exudative age-related macular degeneration in a Chinese cohort," Neuroscience Letters, vol. 488, no. 3, pp. 283-287, 2011.

[43] B. Vojniković, S. Synek, V. Mićović, M. Teležar, and Z. Linšak, "Epidemiological study of sun exposure and visual field damage in children in Primorsko-Goranska County-the risk factors of earlier development of macular degeneration," Collegium Antropologicum, vol. 34, supplement 2, pp. 57-59, 2010.

[44] F. Jonasson, A. Arnarsson, H. Sasaki, T. Peto, K. Sasaki, and A. C. Bird, "The prevalence of age-related maculopathy in Iceland: reykjavik eye study," Archives of Ophthalmology, vol. 121, no. 3, pp. 379-385, 2003.

[45] A. Ostenfeld-Åkerblom, "Age-related macular degeneration in Inuit," Acta Ophthalmologica Scandinavica, vol. 77, no. 1, pp. 76-78, 1999.

[46] P. Mitchell, W. Smith, K. Attebo, and Jie Jin Wang, "Prevalence of age-related maculopathy in Australia: the blue mountains eye study," Ophthalmology, vol. 102, no. 10, pp. 1450-1460, 1995.

[47] L. Xu, S. Wang, Y. Li, and J. B. Jonas, "Retinal vascular abnormalities and prevalence of age-related macular degeneration in adult Chinese: the Beijing Eye study," American Journal of Ophthalmology, vol. 142, no. 4, pp. 688-689, 2006.

[48] S. Synek, B. Vojniković, and D. Pahor, "Epidemiology and quality of life of patients with age-related macular degeneration," Collegium Antropologicum, vol. 34, supplement 2, pp. 25-28, 2010.

[49] B. Muñoz, R. Klein, J. Rodriguez, R. Snyder, and S. K. West, "Prevalence of age-related macular degeneration in a population-based sample of Hispanic people in Arizona: proyecto VER," Archives of Ophthalmology, vol. 123, no. 11, pp. 15751580, 2005.

[50] B. Muñoz, R. Klein, J. Rodriguez, R. Snyder, and S. K. West, "Prevalence of age-related macular degeneration in a population-based sample of Hispanic people in Arizona: proyecto VER," Archives of Ophthalmology, vol. 123, no. 11, pp. 15751580, 2005.

[51] U. Chakravarthy, T. Y. Wong, A. Fletcher et al., "Clinical risk factors for age-related macular degeneration: a systematic review and meta-analysis," BMC Ophthalmology, vol. 10, no. 1, article 31, 2010.
[52] R. D. Sperduto, L. A. Yannuzzi, J. A. Sorenson et al., "Risk factors for neovascular age-related macular degeneration," Archives of Ophthalmology, vol. 110, no. 12, pp. 1701-1708, 1992.

[53] A. F. Cruess, G. Zlateva, A. M. Pleil, and B. Wirostko, "Photodynamic therapy with verteporfin in age-related macular degeneration: a systematic review of efficacy, safety, treatment modifications and pharmacoeconomic properties," Acta Ophthalmologica, vol. 87, no. 2, pp. 118-132, 2009.

[54] J. Mataix, M. Carmen Desco, E. Palacios, M. Garcia-Pous, and A. Navea, "Photodynamic therapy for age-related macular degeneration treatment: epidemiological and clinical analysis of a long-term study," Ophthalmic Surgery Lasers and Imaging, vol. 40, no. 3, pp. 277-284, 2009.

[55] K. Sonmez, P. A. Sonmez, S. S. Ozkan, and L. S. Atmaca, “Oneyear outcomes of less frequent bevacizumab in age-related macular degeneration," Retina, vol. 31, no. 4, pp. 645-653, 2011.

[56] K. D. Kovacs, M. T. Quirk, T. Kinoshita et al., "A retrospective analysis of triple combination therapy with intravitreal bevacizumab, posterior sub-tenon's triamcinolone acetonide, and low-fluence verteporfin photodynamic therapy in patients with neovascular age-related macular degeneration," Retina, vol. 31, no. 3, pp. 446-452, 2011.

[57] J. B. Jonas, Y. Tao, and F. C. Schlichtenbrede, "Intravitreal bevacizumab for exudative age-related macular degeneration in clinical practice," Journal of Ocular Pharmacology and Therapeutics, vol. 27, no. 5, pp. 467-470, 2011.

[58] C. Trempe, "Ranibizumab for age-related macular degeneration," New England Journal of Medicine, vol. 364, no. 6, pp. 581-582, 2011.

[59] T. L. Sãrensen and H. Kemp, "Ranibizumab treatment in patients with neovascular age-related macular degeneration and very low vision," Acta Ophthalmologica, vol. 89, no. 1, p. e97, 2011.

[60] C. Schmucker, Y. K. Loke, C. Ehlken et al., "Intravitreal bevacizumab (Avastin) versus ranibizumab (Lucentis) for the treatment of age-related macular degeneration: a safety review," British Journal of Ophthalmology, vol. 95, 308317 pages, 2010.

[61] A. Chiang and C. D. Regillo, "Preferred therapies for neovascular age-related macular degeneration," Current Opinion in Ophthalmology, vol. 22, no. 3, pp. 199-204, 2011.

[62] M. H. Berrocal, M. L. Lewis, and H. W. Flynn, "Variations in the clinical course of submacular hemorrhage," American Journal of Ophthalmology, vol. 122, no. 4, pp. 486-493, 1996.

[63] J. L. Borrillo and C. D. Regillo, "Treatment of subretinal hemorrhages with tissue plasminogen activator," Current Opinion in Ophthalmology, vol. 12, no. 3, pp. 207-211, 2001.

[64] C. I. Falkner, H. Leitich, F. Frommlet, P. Bauer, and S. Binder, "The end of submacular surgery for age-related macular degeneration? A meta-analysis," Graefe's Archive for Clinical and Experimental Ophthalmology, vol. 245, no. 4, pp. 490-501, 2007.

[65] P. V. Algvere and S. Seregard, "Age-related maculopathy: pathogenetic features and new treatment modalities," Acta Ophthalmologica Scandinavica, vol. 80, no. 2, pp. 136-143, 2002.

[66] P. A. Campochiaro, "Retinal and choroidal neovascularization," Journal of Cellular Physiology, vol. 184, no. 3, pp. 301310, 2000.

[67] N. S. Dejneka, A. Auricchio, A. M. Maguire et al., "Pharmacologically regulated gene expression in the retina following transduction with viral vectors," Gene Therapy, vol. 8, no. 6, pp. 442-446, 2001. 
[68] D. W. Dawson, O. V. Volpert, P. Gillis et al., "Pigment epithelium-derived factor: a potent inhibitor of angiogenesis," Science, vol. 285, no. 5425, pp. 245-248, 1999.

[69] N. M. Holekamp, N. Bouck, and O. Volpert, "Pigment epithelium-derived factor is deficient in the vitreous of patients with choroidal neovascularization due to age-related macular degeneration," American Journal of Ophthalmology, vol. 134, no. 2, pp. 220-227, 2002.

[70] E. J. Duh, H. S. Yang, I. Suzuma et al., "Pigment epitheliumderived factor suppresses ischemia-induced retinal neovascularization and VEGF-induced migration and growth," Investigative Ophthalmology and Visual Science, vol. 43, no. 3, pp. 821-829, 2002.

[71] T. K. MacLachlan, M. Lukason, M. Collins et al., "Preclinical safety evaluation of AAV2-sFLT01- A gene therapy for agerelated macular degeneration," Molecular Therapy, vol. 19, no. 2, pp. 326-334, 2010.

[72] M. Honda, T. Sakamoto, T. Ishibashi, H. Inomata, and H. Ueno, "Experimental subretinal neovascularization is inhibited by adenovirus-mediated soluble VEGF/flt-1 receptor gene transfection: a role of VEGF and possible treatment for SRN in age-related macular degeneration," Gene Therapy, vol. 7, no. 11, pp. 978-985, 2000.

[73] H. L. Kong, D. Hecht, W. Song et al., "Regional suppression of tumor growth by in vivo transfer of a cDNA encoding a secreted form of the extracellular domain of the flt- 1 vascular endothelial growth factor receptor," Human Gene Therapy, vol. 9, no. 6, pp. 823-833, 1998. 


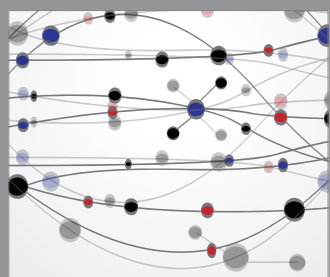

The Scientific World Journal
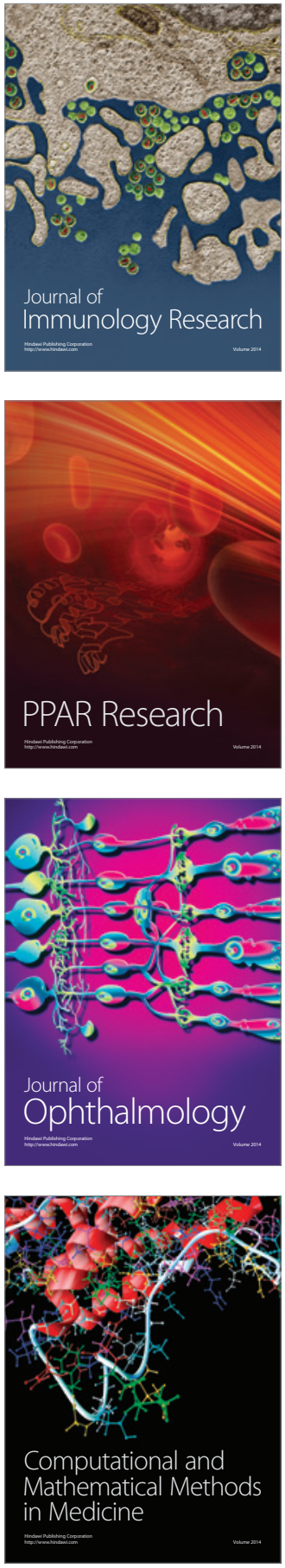

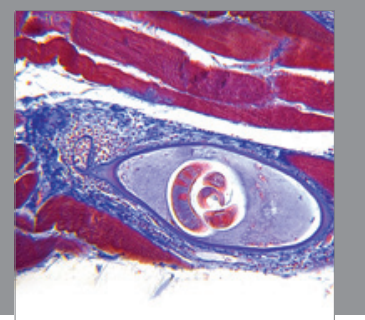

Gastroenterology

Research and Practice
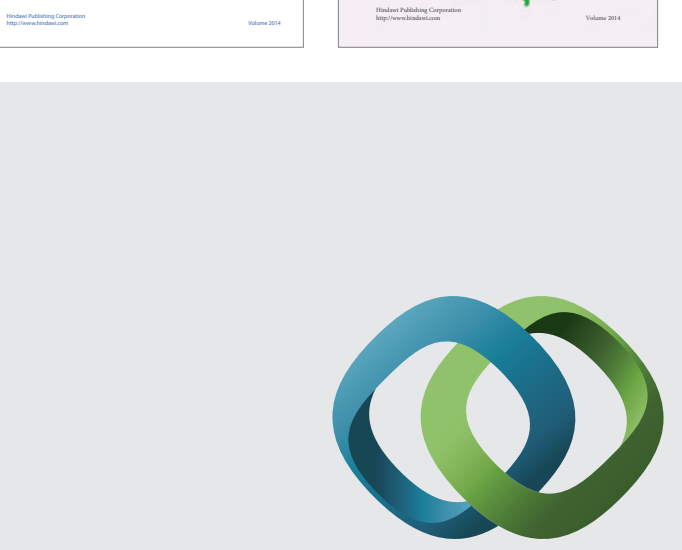

\section{Hindawi}

Submit your manuscripts at

http://www.hindawi.com
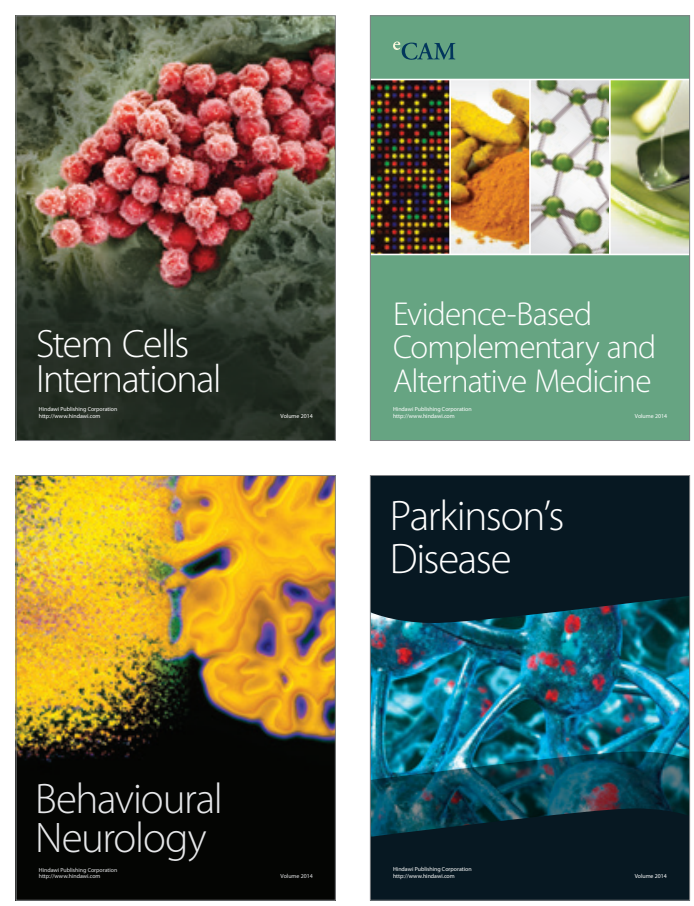

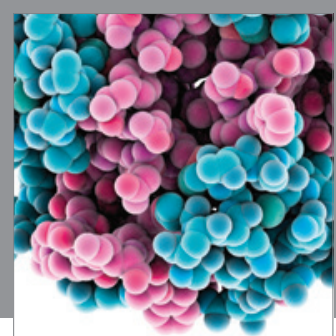

Journal of
Diabetes Research

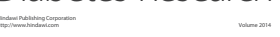

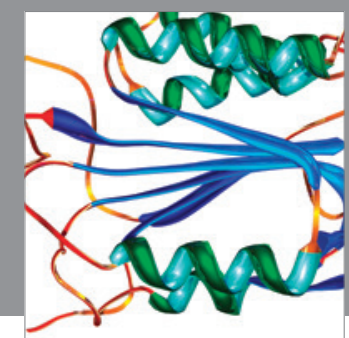

Disease Markers
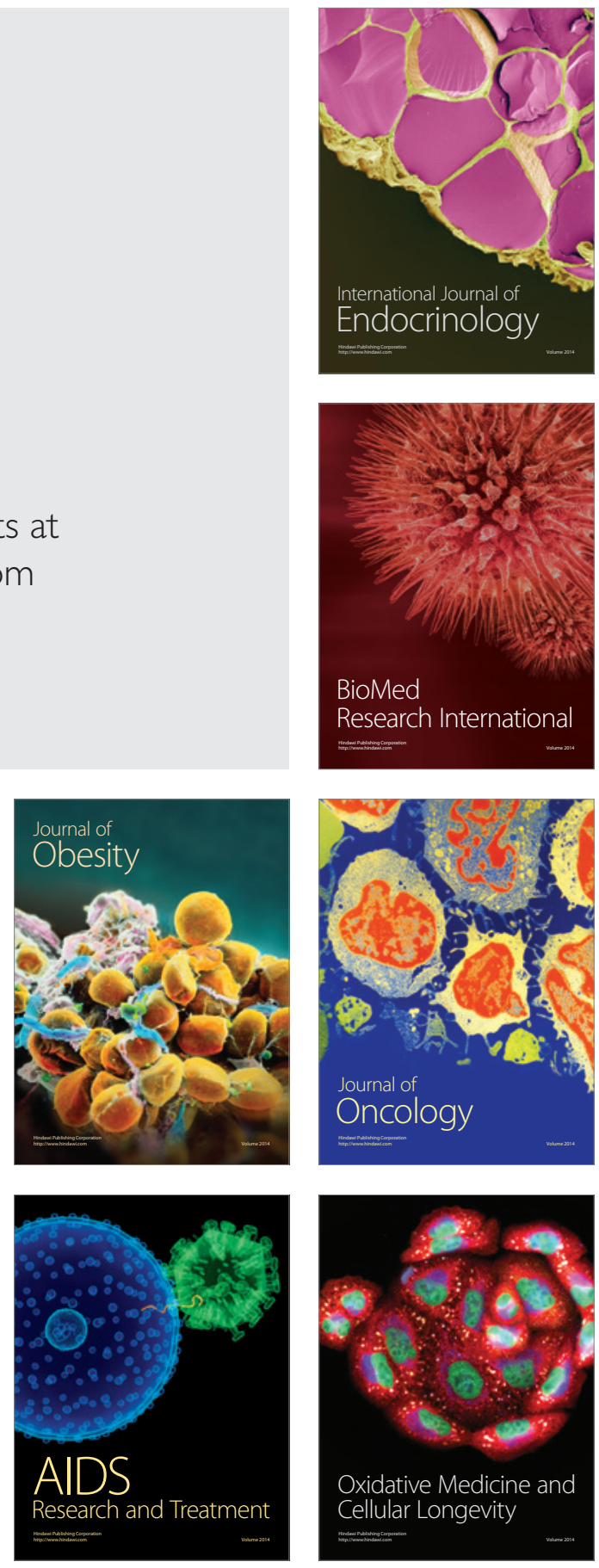